\title{
Legendrian submanifolds with Hamiltonian isotopic symplectizations
}

\author{
Sylvain CouRTE
}

\begin{abstract}
In any closed contact manifold of dimension at least 11 , we construct examples of closed Legendrian submanifolds which are not diffeomorphic but whose Lagrangian cylinders in the symplectization are Hamiltonian isotopic.
\end{abstract}

$53 \mathrm{D} 10$

\section{Introduction}

Let $(M, \xi)$ be a contact manifold ( $\xi$ is cooriented) and denote by $S M$ its symplectization, ie the set of covectors in $T^{*} M$ whose kernel is equal (as a cooriented hyperplane) to $\xi$; it comes with a natural projection $\pi: S M \rightarrow M$, which is a principal $\mathbb{R}$-bundle (the $\mathbb{R}$-action is given by multiplying covectors by $e^{t}$ for $t \in \mathbb{R}$ ). To any Legendrian submanifold $\Lambda \subseteq M$, there corresponds its symplectization $S \Lambda=\pi^{-1}(\Lambda)$ which is a Lagrangian submanifold diffeomorphic to $\mathbb{R} \times \Lambda$. Any $\mathbb{R}$-equivariant Hamiltonian isotopy of $S M$ that takes $S \Lambda$ to $S \Lambda^{\prime}$ induces a contact isotopy of $M$ that takes $\Lambda$ to $\Lambda^{\prime}$. However, if we forget about $\mathbb{R}$-equivariance, we are lead to consider the following question:

Question If $S \Lambda$ and $S \Lambda^{\prime}$ are Hamiltonian isotopic, does it follow that $\Lambda$ and $\Lambda^{\prime}$ are Legendrian isotopic?

This is a relative version of the question of whether contact manifolds with exact symplectomorphic symplectizations are necessarily contactomorphic. The latter question was answered negatively by the author in [3], and we explain in this paper that the same phenomenon arises in this case.

Theorem 1.1 In any closed contact manifold $(M, \xi)$ of dimension $2 n-1 \geqslant 11$, there exist closed Legendrian submanifolds which are not diffeomorphic but whose symplectizations are Hamiltonian isotopic. 
This theorem will follow from a general construction using Lagrangian h-cobordisms and a Mazur-trick argument. An essential ingredient in the proof is the notion of flexible Lagrangian cobordisms recently introduced by Eliashberg, Ganatra and Lazarev in [7]. We need the assumption $2 n-1 \geqslant 11$ because it allows us to apply the s-cobordism theorem to Lagrangian h-cobordisms.

Remark 1.2 Even if we further assume that the Legendrian submanifolds are diffeomorphic, the answer to the question above is still negative. Indeed, we will construct in Section 4 two closed Legendrian submanifolds in $\mathbb{R}^{15}$ which are smoothly isotopic and have Hamiltonian isotopic symplectizations but which are not Legendrian isotopic. Corresponding examples were described by the author in [4, Section 3] for the absolute case.

\section{Exact Lagrangian cobordisms and the Mazur trick}

Let $(M, \xi)$ be a connected contact manifold; recall that its symplectization $S M$ is equipped with a canonical Liouville vector field $X_{\text {can }}$ and a canonical Liouville form $\lambda_{\text {can }}$ (the restrictions of those of $\left.T^{*} M\right)$, and that a contact form for $(M, \xi)$ is a section of the bundle $S M \rightarrow M$. We denote by $S M \geqslant \alpha$ the subset of $S M$ above the section $\alpha$ and use obvious notations for similar subsets of $S M$ or subsets of a Lagrangian cylinder $S \Lambda$.

Definition 2.1 An exact Lagrangian cobordism in $S M$ is a connected Lagrangian submanifold $L \subseteq S M$ such that there exist two sections $\alpha_{-}$and $\alpha_{+}$of $S M$, with $\alpha_{-}<\alpha_{+}$at each point of $M$, with the following properties:

(1) There exist two closed Legendrian submanifolds $\Lambda_{-}$and $\Lambda_{+}$such that

$$
L \cap S M^{\geqslant \alpha_{+}}=S \Lambda_{+}^{\geqslant \alpha_{+}} \text {and } L \cap S M^{\leqslant \alpha_{-}}=S \Lambda_{-}^{\leqslant \alpha_{-}} .
$$

(2) The region $L \cap S M^{\left[\alpha_{-}, \alpha_{+}\right]}$is a compact cobordism from $\Lambda_{-}$to $\Lambda_{+}$(without any other boundary).

(3) Denoting $i: L \rightarrow S M$ the inclusion, there exists a function $g: L \rightarrow \mathbb{R}$ with $i^{*} \lambda_{\text {can }}=\mathrm{d} g$ which is constant on $L \cap S M^{\geqslant \alpha_{+}}$and on $L \cap S M^{\leqslant \alpha_{-}}$.

If $\Lambda_{-}$is empty, we say that $L$ is an exact Lagrangian filling.

Definition 2.2 Let $L$ be an exact Lagrangian submanifold in $S M$. A Liouville vector field $X$ in $S M$ is said to be adapted to $L$ if it is tangent to $L$ and can be written $X=X_{\text {can }}+X_{g}{ }^{1}$ with $g$ constant on $S M \geqslant \alpha_{+}$and on $S M^{\leqslant \alpha_{-}}$for some sections $\alpha_{-}$ and $\alpha_{+}$of $S M$.

${ }^{1}$ The Hamiltonian vector field $X_{g}$ is defined by $\left.X_{g}\right\lrcorner \omega=-\mathrm{d} g$. 
Adapted Liouville vector fields can always be constructed: extend the function $g$ given in Definition 2.1 to $S M$ so that it is constant on $S M \geqslant \alpha_{+}$and on $S M^{\leqslant} \alpha_{-}$and set $X=X_{\text {can }}+X_{g}$.

Definition 2.3 A symplectomorphism $\phi: S M \rightarrow S M$ is said to be contact at infinity if there exist two sections $\alpha_{-}$and $\alpha_{+}$of $S M$ such that in $S M^{\leqslant \alpha_{-}}$(resp. in $\left.S M^{\geqslant} \alpha_{+}\right), \phi$ coincides with the lift of a contactomorphism $\phi_{-}$(resp. $\phi_{+}$). Such symplectomorphisms form a group that we denote by $\mathcal{G}$. The subgroup defined by $\left\{\phi_{+}=\mathrm{id}, \phi_{-}=\mathrm{id}\right\}\left(\right.$ resp. $\left.\left\{\phi_{-}=\mathrm{id}\right\}\right)$ will be denoted by $\mathcal{G}_{\partial}$ (resp. $\left.\mathcal{G}_{\partial_{-}}\right)$. An element of $\mathcal{G}_{\partial_{-}}$is also called a symplectic pseudoisotopy of $M$; see [1, Section 14.5]. We also denote by $\mathcal{H}$ the connected component of the identity in $\mathcal{G}_{\partial}$.

Note that an element $\phi$ of $\mathcal{G}$ is automatically exact; that is, $\phi^{*} \lambda_{\text {can }}=\lambda_{\text {can }}+\mathrm{d} k$ for some function $k: S M \rightarrow \mathbb{R}$ which is constant in $S M^{\geqslant \alpha_{+}}$and in $S M^{\leqslant \alpha_{-}}$for some sections $\alpha_{-}$and $\alpha_{+}$of $S M$. In particular, if $L$ is an exact Lagrangian cobordism in $S M$, then so is $\phi(L)$.

Definition 2.4 Two exact Lagrangian cobordisms $L$ and $L^{\prime}$ in $S M$ are said to be equivalent (which we denote $L \sim L^{\prime}$ ) if there exists an element $\phi$ of $\mathcal{H}$ (see Definition 2.3) such that $L^{\prime}=\phi(L)$.

Exact Lagrangian cobordisms can be composed: given such $\left(L ; \Lambda, \Lambda^{\prime}\right)$ and $\left(L^{\prime} ; \Lambda^{\prime}, \Lambda^{\prime \prime}\right)$, we have sections $\alpha$ and $\alpha^{\prime}$ such that $L \cap S M^{\geqslant \alpha}=S \Lambda^{\prime \geqslant \alpha}$ and $L^{\prime} \cap S M^{\leqslant \alpha^{\prime}}=S \Lambda^{\prime \leqslant \alpha^{\prime}}$. If we can find such sections with $\alpha<\alpha^{\prime}$, then $L$ and $L^{\prime}$ can naturally be glued because they both coincide with $S \Lambda^{\prime}$ in $S M^{\left[\alpha, \alpha^{\prime}\right]}$; now observe that we can always achieve this condition by pushing up $L^{\prime}$ along the flow of $X_{\text {can }}$. We denote by $L \odot L^{\prime}$ the resulting exact Lagrangian cobordism. This composition operation satisfies the following properties:

(1) The equivalence class of $L \odot L^{\prime}$ is independent of choices and depends only on the equivalence classes of $L$ and $L^{\prime}$.

(2) $L \odot S \Lambda^{\prime} \sim L$ and $S \Lambda \odot L \sim L$.

(3) The composition is associative on equivalence classes; that is, $L \odot\left(L^{\prime} \odot L^{\prime \prime}\right) \sim$ $\left(L \odot L^{\prime}\right) \odot L^{\prime \prime}$.

(4) Given a sequence $\left(L_{i} ; \Lambda_{i}, \Lambda_{i+1}\right)$ for $i \in \mathbb{Z}$ of exact Lagrangian cobordisms, we can construct the infinite composition $\bigodot_{i \in \mathbb{Z}} L_{i}$ : this is a properly embedded Lagrangian submanifold (not necessarily an exact Lagrangian cobordism) whose Hamiltonian isotopy class (not with compact support) is independent of choices and only depends on the equivalence class of each $L_{i}$. Moreover, one can introduce parentheses in such an infinite composition as we please. 
Definition 2.5 An exact Lagrangian cobordism $\left(L ; \Lambda, \Lambda^{\prime}\right)$ is said to be invertible if there exists another exact Lagrangian cobordism $\left(L^{\prime} ; \Lambda^{\prime}, \Lambda\right)$ such that $L \odot L^{\prime} \sim S \Lambda$ and $L^{\prime} \odot L \sim S \Lambda^{\prime}$.

To show invertibility of some exact Lagrangian cobordism, we will always construct right inverses only and apply the following proposition.

Proposition 2.6 Let $L, L^{\prime}$ and $L^{\prime \prime}$ be exact Lagrangian cobordisms with $L \odot L^{\prime} \sim S \Lambda$ and $L^{\prime} \odot L^{\prime \prime} \sim S \Lambda^{\prime}$. Then $L$ is invertible.

Proof By associativity of composition, we have

$$
L \sim L \odot\left(L^{\prime} \odot L^{\prime \prime}\right) \sim\left(L \odot L^{\prime}\right) \odot L^{\prime \prime} \sim L^{\prime \prime} .
$$

Proposition 2.7 Let $\Lambda$ and $\Lambda^{\prime}$ be closed Legendrian submanifolds of a closed contact manifold $(M, \xi)$. The following assertions are equivalent:

(1) $S \Lambda$ and $S \Lambda^{\prime}$ are Hamiltonian isotopic (by an isotopy which is not necessarily compactly supported).

(2) There exists an invertible exact Lagrangian cobordism $\left(L ; \Lambda, \Lambda^{\prime}\right)$.

Proof $(1) \Longrightarrow(2)$ Let $H_{t}: S M \rightarrow \mathbb{R}$ be a Hamiltonian generating an isotopy $\phi_{t}$ $(t \in[0,1])$ of $S M$ such that $\phi_{0}=$ id and $\phi_{1}(S \Lambda)=S \Lambda^{\prime}$. We pick four sections $\alpha_{1}<\alpha_{2}<\alpha_{3}<\alpha_{4}$ and two functions $\rho, \rho^{\prime}: S M \rightarrow[0,1]$ with the following properties:

- $\rho=1$ in $S M^{\geqslant \alpha_{2}}$ and $\rho=0$ in $S M^{\leqslant \alpha_{1}}$,

- $\rho^{\prime}=1$ in $S M \leqslant \alpha_{3}$ and $\rho^{\prime}=0$ in $S M \geqslant \alpha_{4}$.

We respectively denote by $\psi_{t}, \psi_{t}^{\prime}$ and $\theta_{t}$ the Hamiltonian isotopies generated by $\rho H_{t}, \rho^{\prime} H_{t}$ and $\rho \rho^{\prime} H_{t}$ (these are all well defined for $t \in[0,1]$ ). Then $L=\psi_{1}(S \Lambda)$ and $L^{\prime}=\psi_{1}^{\prime}(S \Lambda)$ are exact Lagrangian cobordisms from $\Lambda$ to $\Lambda^{\prime}$ and from $\Lambda^{\prime}$ to $\Lambda$, respectively. Moreover, if we chose $\alpha_{3} / \alpha_{2}$ sufficiently big, then $L \odot L^{\prime}$ sits naturally in $S M$ as $\theta_{1}(S \Lambda)$ and is equivalent to $S \Lambda$ (via the isotopy $\theta_{t}$ ). We can likewise construct a right inverse for $L^{\prime}$, and we conclude using Proposition 2.6.

(2) $\Longrightarrow(1)$ Let $\left(L^{\prime} ; \Lambda^{\prime}, \Lambda\right)$ be an inverse for $\left(L ; \Lambda, \Lambda^{\prime}\right)$ and consider the infinite composition

$$
L_{\infty}=\cdots \odot L \odot L^{\prime} \odot L \odot L^{\prime} \odot \cdots
$$

By introducing parentheses in two different ways, $\left(L \odot L^{\prime}\right)$ versus $\left(L^{\prime} \odot L\right)$, in the above expression, we get that $L_{\infty}$ is Hamiltonian isotopic to $S \Lambda$ as well as to $S \Lambda^{\prime}$. 
Remark 2.8 It follows from Proposition 2.7 that no invariant of closed Legendrian submanifolds which is functorial with respect to exact Lagrangian cobordisms can distinguish between $\Lambda$ and $\Lambda^{\prime}$. This applies in particular to Legendrian contact homology introduced in [8, Section 2.8]; see also [5] and [6].

Here is a first class of examples of invertible Lagrangian cobordisms that will appear in the next section.

Proposition 2.9 Let $(M, \xi)$ be a closed contact manifold, $\Lambda$ a closed Legendrian submanifold of $M$, and $\phi: S M \rightarrow S M$ a symplectic pseudoisotopy (see Definition 2.3). Then $\phi(S \Lambda)$ is an invertible exact Lagrangian cobordism in $S M$.

Proof There exists some section $\alpha$ of $S M$ such that $\phi$ coincides with the lift of some contactomorphism $\phi_{+}$in $S M^{\geqslant} \geqslant \alpha_{+}$. We have that $L=\phi(S \Lambda)$ is an exact Lagrangian cobordism in $S M$ from $\Lambda$ to $\Lambda^{\prime}=\phi_{+}(\Lambda)$. Besides, $L^{\prime}=\phi^{-1}\left(S \Lambda^{\prime}\right)$ is an exact Lagrangian cobordism from $\Lambda^{\prime}$ to $\Lambda$. Let $\psi_{t}$ be the flow of $X_{\text {can }}$, and let $\phi_{t}=\psi_{-t} \circ \phi \circ \psi_{t}$. We have that $\phi^{-1} \circ \phi_{t}$ is a smooth path in $\mathcal{G}$ starting from id and that, for $t>0$ large enough, $\phi^{-1} \circ \phi_{t}(S \Lambda)$ is a realization of the composition $L \odot L^{\prime}$. So we get $L \odot L^{\prime} \sim S \Lambda$. Likewise, we can produce a right inverse for $L^{\prime}$ and conclude by Proposition 2.6.

We end this section with the following result, whose proof is almost the same as that of Proposition 2.7 except that we use the Mazur trick only in the positive end.

Proposition 2.10 Let $(M, \xi)$ be a closed contact manifold, and let $F$ and $F^{\prime}$ be two exact Lagrangian fillings in $S M$ (see Definition 2.1). The following assertions are equivalent:

(1) $F$ and $F^{\prime}$ are Hamiltonian isotopic (by an isotopy which is not necessarily compactly supported).

(2) There exists an invertible exact Lagrangian cobordism $L$ such that $F \odot L \sim F^{\prime}$.

Our goal now is to construct invertible exact Lagrangian cobordisms which are not diffeomorphic to cylinders.

\section{Flexible Lagrangian h-cobordisms}

Let $(M, \xi)$ be a contact manifold of dimension $2 n-1 \geqslant 5$. 
Definition 3.1 [7] An exact Lagrangian cobordism $L \subseteq S M$ is called regular if there exist an adapted Liouville vector field $X$ (see Definition 2.2) and a proper Morse function $f: S M \rightarrow \mathbb{R}$ for which $X$ is a pseudogradient. Moreover, if there exists such an adapted pair $(f, X)$ for which $f$ is excellent (all critical values are distinct) and the attaching spheres of critical points of index $n$ are loose (see [15]) in the complement of $L$, then $L$ (as well as the pair $(f, X))$ is said to be flexible.

Note that the critical points of $f \mid L$ are necessarily critical points of $f$, and in the flexible case, there cannot be any critical point of index $n$ on $L$. The definition can obviously be extended to Lagrangian cobordisms into arbitrary flexible Weinstein cobordisms.

Recall that an $h$-cobordism is a cobordism which deformation retracts on its bottom boundary as well as on its top boundary. According to the s-cobordism theorem (see [12]), h-cobordisms from a given closed manifold $M$ of dimension $\geqslant 5$ are classified up to diffeomorphism relative to $M$ by so-called Whitehead torsion, an invariant which takes values in the Whitehead group $\mathrm{Wh}(M)$ of $M$ (it actually depends only on $\left.\pi_{1} M\right)$. Essentially, since each element in a group has an inverse, h-cobordisms of dimension $\geqslant 6$ are invertible for the composition of cobordisms; see [16].

Theorem 3.2 Let $(M, \xi)$ be a closed contact manifold of dimension $\geqslant 11$.

(1) Let $\Lambda$ be a closed Legendrian submanifold in $M$, and $\left(L ; \Lambda, \Lambda^{\prime}\right)$ an $h$-cobordism. Then $L$ can be embedded in $S M$ as a flexible Lagrangian cobordism starting from $\Lambda$.

(2) Any flexible Lagrangian h-cobordism in $S M$ is invertible (as an exact Lagrangian cobordism).

We need a couple of intermediate results. The following lemma is proved in [7, Proposition 2.5].

Lemma 3.3 For any regular Lagrangian cobordism $L$ together with an adapted pair $(f, X)$, we can find a homotopy $\left(f_{t}, X_{t}\right)$ of adapted pairs such that $\left(f_{0}, X_{0}\right)=(f, X)$, and for all critical point of $f_{1}$ on $L$, the index is the same for $f_{1}$ and $f_{1} \mid L$. Moreover, if $\operatorname{dim} L \geqslant 3$ and if $\left(f_{0}, X_{0}\right)$ is flexible, we can require $\left(f_{t}, X_{t}\right)$ to be flexible for all $t$.

The following theorem is a relative analogue of [1, Corollary 14.2] and may be of independent interest.

Theorem 3.4 Let $(M, \xi)$ be a contact manifold of dimension $2 n-1 \geqslant 5$. If $\left(L ; \Lambda, \Lambda^{\prime}\right)$ is a flexible Lagrangian cobordism in $S M$ which is diffeomorphic to $\Lambda \times[0,1]$ (with cylindrical ends attached), then it admits an adapted pair without critical points. 
Proof We start with a flexible adapted pair $(f, X)$.

By Lemma 3.3, we can assume that the critical points on $L$ have the same index for $f \mid L$ and $f$.

Since there are no $X$-trajectories going from critical points outside of $L$ to critical points on $L$, we can reorder the critical values so that the critical points on $L$ lie below all the others (by repeated applications of [13, Lemma 2.1], starting with the lower critical point on $L$ ). After this modification, there is a level set $\{f=c\}$ which separates critical points outside of $L$ from critical points on $L$.

Since $L$ is a cylinder, $L \cap\{f \leqslant c\}$ admits a function $h$ without critical points together with a pseudogradient $Y$, and we can take them to coincide with the restrictions of $(f, X)$ near $f^{-1}(c)$ and below some section $\alpha_{-}$of $S M$. We extend $h$ to an adapted pair $(\hat{h}, \hat{Y})$ on a neighborhood of $L$ in a standard way (see [1, Lemma 12.8]), and using [1, Lemma 12.10], we modify $(f, X)$ near $L$ by interpolation so that it coincides with $(\hat{h}, \hat{Y})$ near $L$. Now $(f, X)$ has only critical points outside of $L$, and it is still flexible because nothing has changed above the level set $\{f=c\}$.

It remains to cancel the critical points outside of $L$, and we will do it by a path of adapted pairs which is constant near $L$. We first need to prove that it is smoothly possible (that is, without the condition that the pseudogradient is Liouville). For that, we can follow the proof of the s-cobordism theorem (as in [12]):

(1) Cancel critical points of index 0 with some critical points of index 1.

(2) Trade critical points of index $i$ for critical points of index $i+2$, until there only remain critical points of indices $n-1$ and $n$.

(3) Cancel together critical points of indices $n-1$ and $n$.

Recall that every $X$-trajectory between critical points is disjoint from $L$, so $L$ appears in each level set of $f$ as a smooth submanifold of codimension $n$ (which is at least 3 ) and is disjoint from the stable and unstable manifolds of all critical points. The main point to notice is that the isotopies of the attaching spheres needed to arrange cancellation positions can be done in the complement of $L$ because they can be localized near Whitney 2 -disks which are generically disjoint from $L$. Therefore, we can proceed through all the steps above keeping $(f, X)$ fixed near $L$.

Finally, as in the proof of Theorem 14.9 in [1], we upgrade the deformation of $(f, X)$ to a deformation through adapted pairs (that is, with Liouville pseudogradient). Since $(f, X)$ is flexible, the attaching spheres of critical points are either subcritical or loose in the complement of $L$, so the same proof works verbatim and provides the required path of adapted pairs, fixed near $L$, which cancels all critical points of $(f, X)$. 
Proof of Theorem 3.2 (1) Recall that any h-cobordism of dimension at least 6 can be presented with a Morse function having only critical points of indices 2 and 3; see [12]. We first construct a flexible ${ }^{2}$ Weinstein cobordism $\left(W ; M, M^{\prime}\right)$ containing a flexible Lagrangian cobordism $\left(L ; \Lambda, \Lambda^{\prime}\right)$ by attaching Weinstein handles of indices 2 and 3 on $\Lambda$. Denoting by $\tau \in \operatorname{Wh}(L)$ the Whitehead torsion of $L$, we note that the ambient cobordism $W$ is also an h-cobordism and that its torsion is $i(\tau)$, where $i: \mathrm{Wh}(\Lambda) \rightarrow \mathrm{Wh}(M)$ is the map induced by inclusion. We now attach handles of indices 2 and 3 on top of $M^{\prime}$ away from $\Lambda^{\prime}$ to produce a flexible Weinstein hcobordism $W^{\prime}$ with torsion $-i(\tau) \in \mathrm{Wh}\left(M^{\prime}\right)$ (we identify $\mathrm{Wh}(M) \simeq \mathrm{Wh}\left(M^{\prime}\right)$ via the homotopy equivalence induced by $W$ ). The Lagrangian $L$ can be continued inside of $W^{\prime}$ by composing with the Lagrangian cylinder $S \Lambda^{\prime}$. The composition $W \odot W^{\prime}$ is a flexible Weinstein cobordism, and it is diffeomorphic to $M \times[0,1]$ since its Whitehead torsion vanishes. We can therefore cancel all the handles and show that $W \odot W^{\prime}$ is equivalent to $S M$ relative to the negative boundary; see [1, Corollary 14.2]. Thus $L$ now sits as a flexible Lagrangian cobordism in $S M$.

(2) Let $\left(L_{1}^{\prime} ; \Lambda^{\prime}, \Lambda\right)$ be an inverse cobordism for $\left(L ; \Lambda, \Lambda^{\prime}\right)$. Using the first point, we can embed $L_{1}^{\prime}$ as a flexible Lagrangian cobordism in $S M$. Denote by $\Lambda_{1}$ the positive Legendrian boundary of $L_{1}^{\prime}$; note that it is a priori different from $\Lambda$. Now Theorem 3.4 allows us to find an adapted pair $(f, X)$ without critical points for the composition $L \odot L_{1}^{\prime}$. By sending the trajectories of $X_{\text {can }}$ to that of $X$, we find a symplectic pseudoisotopy $\psi$ of $S M$ (that is, $\psi \in \mathcal{G}_{\partial_{-}}$; see Definition 2.3) that takes $S \Lambda$ to $L \odot L_{1}^{\prime}$. By Proposition 2.9, there is an exact Lagrangian cobordism $L_{2}^{\prime}$ such that $L \odot L_{1}^{\prime} \odot L_{2}^{\prime} \sim S \Lambda$. Note further that $L_{2}^{\prime}$ (as constructed in the proof of Proposition 2.9) admits an adapted pair without critical points, so it is flexible. Hence $L^{\prime}=L_{1}^{\prime} \odot L_{2}^{\prime}$ is a right inverse for $L$, and we can repeat the same argument to produce a right inverse for $L^{\prime}$. The result now follows from Proposition 2.6.

\section{Examples}

An example where $\boldsymbol{\Lambda}$ and $\boldsymbol{\Lambda}^{\prime}$ are not diffeomorphic For $n \geqslant 7$, consider the manifold $\Lambda=\mathrm{L}(4,1) \times \mathrm{T}^{n-4}$. It was proved in [10] that there exists an h-cobordism $\left(L ; \Lambda, \Lambda^{\prime}\right)$ such that $\Lambda^{\prime}$ is not diffeomorphic to $\Lambda$. For the case $n=6$, we can take the manifold $\Lambda=\mathrm{L}(7,1) \times \mathrm{S}^{2}$ which is h-cobordant but not diffeomorphic to $\Lambda^{\prime}=\mathrm{L}(7,2) \times \mathrm{S}^{2}$; see [14]. We claim that $\Lambda$ admits a Legendrian embedding into $\mathbb{R}^{2 n-1}$ endowed with its standard contact structure. Indeed, $\Lambda$ is parallelizable so we can find a Legendrian bundle monomorphism $T \Lambda \rightarrow T \mathbb{R}^{2 n-1}$ and then turn it into

\footnotetext{
${ }^{2}$ It is actually even subcritical: there are no handles of index $n$.
} 
a Legendrian embedding via Gromov's h-principle; see [9, Theorem 16.1.3], and note that a generic Legendrian immersion is an embedding. This Legendrian embedding of $\Lambda$ can be implanted in any contact manifold via a Darboux chart. Theorem 1.1 now follows from Theorem 3.2 and Proposition 2.7.

\section{An example where $\Lambda$ and $\Lambda^{\prime}$ are smoothly isotopic but not Legendrian isotopic} The following construction is similar to that in [4, Section 3], but is slightly more involved.

Consider the closed 7-dimensional manifold $\Lambda=\mathrm{L}(5,1) \times \mathrm{S}^{4}$. Note that $\Lambda$ is parallelizable and that $\pi_{3} \Lambda=\pi_{3} \mathrm{~L}(5,1)=\mathbb{Z}$ (a generator is given by the universal covering map $\left.\mathrm{S}^{3} \rightarrow \mathrm{L}(5,1)\right)$.

Lemma 4.1 (1) There exists an h-cobordism $(L ; \Lambda, \Lambda)$ such that the induced map $f: \Lambda \rightarrow \Lambda$ acts by multiplication by -1 on $\pi_{3} \Lambda$.

(2) No diffeomorphism of $\Lambda$ may act by multiplication by -1 on $\pi_{3} \Lambda$.

Proof (1) There are exactly two homotopy classes of maps $\mathrm{L}(5,1) \rightarrow \mathrm{L}(5,1)$ of degree -1 (these are automatically homotopy equivalences) and they respectively induce multiplication by 2 and -2 on $\pi_{1} L(5,1)=\mathbb{Z} / 5 \mathbb{Z}$; see $[2,29.5]$. We pick such a map and perturb it to an embedding $j: \mathrm{L}(5,1) \rightarrow \mathrm{L}(5,1) \times$ int $\mathrm{D}^{5}$ using Whitney's embedding theorem. The normal bundle of $j$ is trivial because it is stably trivial and has rank greater than the dimension of the base. We can therefore extend $j$ to an embedding $\mathrm{L}(5,1) \times \mathrm{D}^{5} \rightarrow \mathrm{L}(5,1) \times$ int $\mathrm{D}^{5}$ that we still denote by $j$. The region $L=\mathrm{L}(5,1) \times \mathrm{D}^{5} \backslash j\left(\mathrm{~L}(5,1) \times\right.$ int $\left.\mathrm{D}^{5}\right)$ is an h-cobordism from $\Lambda$ to itself; see [14, Lemma 2, page 579]. The map $f: \Lambda \rightarrow \Lambda$ induced by the cobordism $L$ can be defined as $f=r \circ i$, where $i: \Lambda \rightarrow L$ is the inclusion of the negative boundary and $r: L \rightarrow \Lambda$ is a deformation retraction on the positive boundary (the homotopy class of $f$ is independent of choices). Since we started with a map of degree -1 on $\mathrm{L}(5,1)$, we see that $j$ induces multiplication by -1 on $H_{3}\left(\mathrm{~L}(5,1) \times \mathrm{D}^{5} ; \mathbb{Z}\right) \simeq \mathbb{Z}$ as well as on $\pi_{3}\left(\mathrm{~L}(5,1) \times \mathrm{D}^{5}\right) \simeq \mathbb{Z}$ because the Hurewicz homomorphism $\pi_{3} \mathrm{~L}(5,1) \rightarrow$ $H_{3}(\mathrm{~L}(5,1) ; \mathbb{Z})$ is nonzero. It follows from the commutativity up to homotopy of the diagram

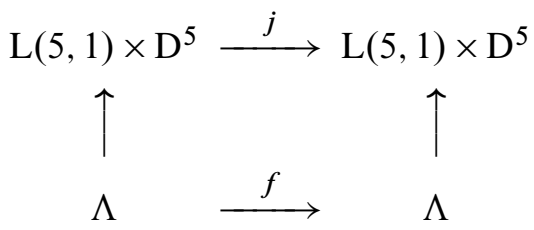

that the map $f$ also induces multiplication by -1 on $\pi_{3} \Lambda$ (the vertical arrows are obvious inclusions). 
(2) If $\psi: \Lambda \rightarrow \Lambda$ was such a diffeomorphism, then the map $\mathrm{L}(5,1) \rightarrow \mathrm{L}(5,1)$, obtained by composing the inclusion of a factor with $\psi$ and then projection, would have degree -1 . But then $\psi$ necessarily acts by multiplication by \pm 2 on $\pi_{1}$, in which case the Whitehead torsion of $\psi$ must be nonzero (see [4, Lemma 3.2]), contradicting the fact that $\psi$ is a diffeomorphism.

Let $(L ; \Lambda, \Lambda)$ be an h-cobordism given by the lemma above. We fix a framing of $\Lambda$ and extend it to a framing of $L$ by using an isomorphism $T L \rightarrow \mathbb{R} \times T \Lambda$ lifting the retraction map $r: L \rightarrow \Lambda$ on the positive boundary. Note that the induced framing of $T \Lambda \times \mathbb{R}$ on the negative boundary, a priori, differs from the given one: it is the image of the given framing by a map $A: \Lambda \rightarrow O(8) \subseteq U(8)$. Recall that any Legendrian immersion $\Lambda \rightarrow \mathbb{R}^{15}$ gives rise to a map $\Lambda \rightarrow U(7)$ well-defined up to homotopy, and Gromov's h-principle (see [9, Theorem 16.1.3]) implies that this classifies Legendrian regular homotopy classes. Given an embedding of $L$ as a Lagrangian cobordism in $S \mathbb{R}^{15}$, we get maps $g: \Lambda \rightarrow U(7), g^{\prime}: \Lambda \rightarrow U(7)$ and $G: L \rightarrow U(8)$ respectively associated to $\partial_{-} L, \partial_{+} L$ and $L$. These maps are related by the formulas

$$
\text { A.s } \circ g \sim G \circ i \text { and } s \circ g^{\prime} \circ r \sim G,
$$

where $\sim$ here means homotopic, $s: U(7) \rightarrow U(8)$ is the stabilization map (note that this is an isomorphism on $\pi_{3}$ ), $r$ and $i$ are defined as in the proof of Lemma 4.1 and the dot denotes multiplication in $U(8)$. In particular, we get $s \circ g^{\prime}$ out of $s \circ g$ :

$$
s \circ g^{\prime} \sim A . s \circ g \circ f^{-1} .
$$

Recall from Bott periodicity that $\pi_{3} U(8) \simeq \mathbb{Z}$. Identifying $\pi_{3} \Lambda$ and $\pi_{3} U(8)$ with $\mathbb{Z}$, the maps induced on $\pi_{3}$ by $s \circ g, s \circ g^{\prime}$ and $A$ are multiplication by integers $b, b^{\prime}$ and $a$, respectively, and the equation above reads

$$
b^{\prime}=a-b .
$$

(Recall that multiplication on $U(8)$ induces addition on $\pi_{3} U(8)$ and that $f$ acts by -1 on $\pi_{3} \Lambda$.)

We now observe that, whatever $a$ is, we can choose $g$ such that $b^{\prime} \neq b$, and therefore, $g^{\prime}$ is not homotopic to $g$. Indeed,

- if $a \neq 0$, we take $g$ to be constant so that $b=0$ and $b^{\prime} \neq 0$;

- if $a=0$, we take $g=\alpha \circ h \circ p_{1}$, where $p_{1}: \Lambda \rightarrow \mathrm{L}(5,1)$ is the projection on the first factor, $h: \mathrm{L}(5,1) \rightarrow \mathrm{S}^{3}$ is a map of degree 1 and $\alpha: \mathrm{S}^{3} \rightarrow U(7)$ corresponds to $1 \in \mathbb{Z}=\pi_{3} U(7)=\pi_{3} U(8)$, so that $b=5$ and $b^{\prime}=-5$. 
The rest of the construction is the same as in the first example above. We take a Legendrian embedding $\phi: \Lambda \rightarrow \mathbb{R}^{15}$ that induces the map $g$ and then use Theorem 3.2 to obtain an embedding of $L$ as a flexible Lagrangian cobordism in $S \mathbb{R}^{15}$ with negative boundary $\phi$. The new Legendrian embedding $\phi^{\prime}: \Lambda \rightarrow \mathbb{R}^{15}$ that we get on the positive boundary of $L$ has a corresponding map to $U(7)$ which is homotopic to $g^{\prime}$, so the Legendrian embeddings $\phi$ and $\phi^{\prime}$ are not homotopic through Legendrian immersions. Moreover, using the second point of Lemma 4.1, we see that this cannot be arranged by composing $\phi^{\prime}$ by a diffeomorphism of $\Lambda$. Hence the (unparametrized) Legendrian submanifolds $\phi(\Lambda)$ and $\phi^{\prime}(\Lambda)$ are not Legendrian isotopic, though they have Hamiltonian isotopic symplectizations (by Theorem 3.2 and Proposition 2.7), and they are smoothly isotopic by Haefliger's embedding theorem (see [11, théorème d'existence b]).

Remark 4.2 The same construction also works for $\mathrm{L}(5,1) \times \mathrm{S}^{2 k}$ for all $k \geqslant 1$, and it is certainly possible to find also examples of this kind in the remaining dimensions.

Acknowledgments I would like to thank Yasha Eliashberg and Tobias Ekholm for encouraging discussions, and also Thomas Kragh and Rémi Crétois for useful discussions concerning the second example in Section 4. I am also grateful for valuable suggestions from the referee. Finally, I acknowledge support from the Knut and Alice Wallenberg Foundation.

\section{References}

[1] K Cieliebak, Y Eliashberg, From Stein to Weinstein and back, Amer. Math. Soc. Colloq. Publ. 59, Amer. Math. Soc., Providence, RI (2012) MR

[2] M M Cohen, A course in simple-homotopy theory, Graduate Texts in Mathematics 10, Springer, New York (1973) MR

[3] S Courte, Contact manifolds with symplectomorphic symplectizations, Geom. Topol. 18 (2014) 1-15 MR

[4] S Courte, Contact manifolds and Weinstein h-cobordisms, J. Symplectic Geom. 14 (2016) 657-669

[5] T Ekholm, Rational symplectic field theory over $\mathbb{Z}_{2}$ for exact Lagrangian cobordisms, J. Eur. Math. Soc. 10 (2008) 641-704 MR

[6] T Ekholm, K Honda, T Kálmán, Legendrian knots and exact Lagrangian cobordisms, J. Eur. Math. Soc. 18 (2016) 2627-2689 MR

[7] Y Eliashberg, S Ganatra, O Lazarev, Flexible Lagrangians, preprint (2015) arXiv

[8] Y Eliashberg, A Givental, H Hofer, Introduction to symplectic field theory, Geom. Funct. Anal. (2000) 560-673 MR 
[9] Y Eliashberg, N Mishachev, Introduction to the $h$-principle, Graduate Studies in Mathematics 48, Amer. Math. Soc., Providence, RI (2002) MR

[10] F T Farrell, W-C Hsiang, $H$-cobordant manifolds are not necessarily homeomorphic, Bull. Amer. Math. Soc. 73 (1967) 741-744 MR

[11] A Haefliger, Plongements différentiables de variétés dans variétés, Comment. Math. Helv. 36 (1961) 47-82 MR

[12] M A Kervaire, Le théorème de Barden-Mazur-Stallings, Comment. Math. Helv. 40 (1965) 31-42 MR

[13] F Laudenbach, A proof of Reidemeister-Singer's theorem by Cerf's methods, Ann. Fac. Sci. Toulouse Math. 23 (2014) 197-221 MR

[14] J Milnor, Two complexes which are homeomorphic but combinatorially distinct, Ann. of Math. 74 (1961) 575-590 MR

[15] E Murphy, Loose Legendrian embeddings in high-dimensional contact manifolds, preprint (2012) arXiv

[16] J R Stallings, On infinite processes leading to differentiability in the complement of a point, from "Differential and Combinatorial Topology (A Symposium in Honor of Marston Morse)" (S S Cairns, editor), Princeton Univ. Press (1965) 245-254 MR

Department of Mathematics, Uppsala University, Box 480, 75106 Uppsala, Sweden

sylvain. courte@math.uu.se

Received: 22 January $2016 \quad$ Revised: 17 March 2016 